\title{
Multi-Agent Rendezvous Problem with Preserving Topology Connectivity
}

\author{
Jiahe Tian ${ }^{\text {a) }}$ \\ Computer College of Guangdong University of Technology, Guangzhou,510000, China. \\ a) Corresponding author: 576152161@qq.com
}

\begin{abstract}
This paper studies a distributed multi-agent rendezvous problem that preserves topology connectivity. Based on the constraint function, the agent's own and its neighbor's current time and last time's condition are used as conditions to judge the agent's movement trend and connectivity quality in the system.After that, the evaluation result is used as one of the basis for adjusting the input of the agent control so as to achieve the purpose of preserving the topology connectivity. Finally, taking the 50 agents distributed uniformly on the plane as an example, the simulation results and performance analysis are given. The results show that under this strategy, the multi-agent system can achieve gradual rendezvous at the same point.
\end{abstract}

Key words: multi-agent system; rendezvous; distribute control; preserving connectivity.

\section{INTRODUCTION}

The rendezvous problem is one of the hotspots of multi-agent research. Ando et al. [1] proposed the multi-robot rendezvous problem for the first time and attracted much attention. Since then, based on different practical applications, people have conducted in-depth research. In order to ensure topology network connectivity between agents, many control methods have been proposed. For example, Cortes et al. [2] used an external algorithm to implement existing connections. DP Spanos and RM Murray [3] used a local connectivity measure to ensure the global connectivity of the network. Feng Xiao et al. [4] proposed a topological connectivity maintenance protocol consisting of distributed control laws in a rectangular area to achieve progressive convergence control of multi-agent systems. With the deepening of research, the artificial potential energy field method is widely used to preserve the connectivity of multi-agent networks, and the potential energy function is one of the applications of the artificial potential energy field method. Meng Ji and Magnus Egerstedt [5] proposed a representative preserving the topologically connected potential energy function control method, when the distance between the agents is close to the sensing radius $r$, the purpose of preserving topology network connectivity is achieved by adjusting the control input of the agent. In order to solve the potential energy function effect that the time lag may cause, Housheng Su et al. [6] introduce a lagged adjustment factor in the design of the potential energy function, construct a class of bounded potential energy function, and make the multi-agent dynamics of the second-order integrator. The connectivity is preserved when there is a time lag in the aggregation. Yuan Fan et al. [7] designed a constraint function that not only preserves the topology connectivity among agents, but also makes the multi-agent control input bounded.

At present, most researches on preserving topology connectivity of multi-agents only depend on the distance between current agents or the number of neighbors that have communication connections, which has certain limitations. Based on the traditional multi-agent rendezvous model, this paper draws on the control idea of the constraint function and evaluates the current connectivity quality based on the state of the multi-agent historical moment itself and neighboring neighbors and assesses the poor connectivity quality based on the evaluation results. The body takes certain measures so as to ensure gradual rendezvous of multiple agents at the same point. 


\section{THEORETICAL BASIS AND PROBLEM DESCRIPTION}

In the multi-agent rendezvous model, it is assumed that the agents have the same sensing range $r$ and the network topology is $G=(V, E)$, where $V=\left(v_{1}, v_{2}, \cdots, v_{n}\right)$ is the set of nodes (i.e., agents in the figure), and $E \in V^{2}$ represents the set of edges consisting of node pairs. And $\left\|x_{i j}\right\|=\left\|x_{i}-x_{j}\right\|$ represents the distance between the agent $i$ and agent $j$. Generally use $A=\left[a_{i j}\right]$ to represent adjacency matrix of graph $G$ Where $a_{i j}=1$ that $(i, j) \in E \wedge\left\|x_{i j}\right\|<r$,otherwise $a_{i j}=0$.:

Take the agent $i$ as an example. In the discrete time, the multi-agent distributed rendezvous protocol is:

$$
x_{i}(t+1)=x_{i}(t)+\varepsilon \sum_{j \in N_{i}} a_{i j}\left(x_{j}(t)-x_{i}(t)\right), t \geq 0
$$

Where $\varepsilon$ is the adjustment factor of the agent used to adjust the adjustment factor for each movement, and $N_{i}$ is used to represent the set of neighbor points within the communication range $r$ of the agent $i$,i.e. $N_{i}=\left\{j \mid\left\|x_{i j}\right\|<r\right\}$.

The agent retains the status at a moment on itself and its neighboring neighborhoods as $x_{i}{ }^{\prime}(t)=x_{i}(t-1), x_{j}^{\prime}(t)=x_{j}(t-1)$.

Definition 1:In the neighbor set $N_{i}$ of the agent $i$, if $\left\|x_{i}(t)-x_{j}{ }^{\prime}(t)\right\|-\left\|x_{i}{ }^{\prime}(t)-x_{j}{ }^{\prime}(t)\right\|<0$ for any neighbor $j$, then the agent $i$ is in the edge position and the quality of the connection is good.

Definition 2:In the neighbor set $N_{i}$ of the agent $i$,if $\left\|x_{i}(t)-x_{j}(t)\right\|>\rho$ and $\left\|x_{i}(t)-x_{j}{ }^{\prime}(t)\right\|-\left\|x_{i}{ }^{\prime}(t)-x_{j}{ }^{\prime}(t)\right\|>0$, then the agent $i$ is in a critical position and the quality of connectivity is poor

Definition 3: For any two agents in the system, if $\left\|x_{j}(t)-x_{i}(t)\right\| \rightarrow 0, \forall i, j \in V \wedge t \rightarrow \infty$, then the multi-agent systems achieve gradual rendezvous at the same point [8].

In the early research of multi-agent problems, the topological network of multi-agent rendezvous is assumed to be always connected. However, in the actual rendezvous process, the connectivity of the initial network does not mean that the topology network is always connected [8]. In order to preserve the topological connectivity of the multi-agent as much as possible during the movement, the current quality of the agent is evaluated, and measures are taken to adjust the multi-agent according to the evaluation results, i.e.

$$
x_{i}(t+1)=x_{i}(t)+\varepsilon \prod_{j \in N_{i}} \varphi_{i}\left(\left\|x_{j}-x_{i}\right\|\right) \sum_{j \in N_{i}} a_{i j}\left(x_{j}(t)-x_{i}(t)\right)
$$

Where $\prod_{j \in N_{i}} \varphi\left(\left\|x_{j}-x_{i}\right\|\right)$ is constraint function based on agent connectivity quality.

\section{AGENT CONNECTIVITY QUALITY ASSESSMENT STRATEGY}

In the traditional multi-agent rendezvous problem, when multiple agents are evenly distributed in a large plane, they often cannot be clustered exactly at the same point, and the rendezvous may occur at multiple points. Therefore, it is important to preserve the connectivity of the network topology of the multi-agent system. In the existing research on multi-agent aggregation, most people use artificial potential energy fields to preserve topological connectivity.

The agent only depends on the current time itself and neighbor information measured by the agent and cannot accurately assess its position in the multi-agent system. Although the current movement trend of the agent can be assessed by the change in distance from the neighbor at the previous moment, it may not apply to some situations. Therefore, a new multi-agent rendezvous algorithm based on historical state to assess connectivity quality is proposed to preserve topology connectivity between agents. 


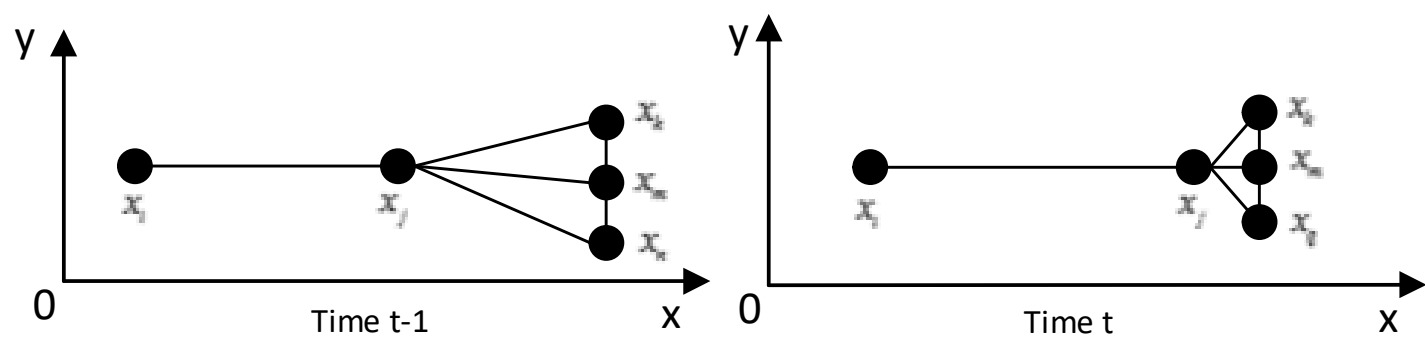

FIGURE 1. Agent distance changes

As shown in Fig.1,although the distance between the agent $i$ and the agent $j$ increases, the agent $i$ moves in the direction of the agent $j$.Therefore, according to the distance between agent $i$ at $t$ and neighbor agent $j$ at time $t-1$ and distance difference between agents $i$ at $t$ and $j$ at $t$-1,judging the movement of agents. As defined in Definition 1 , the agent $i$ is at the edge.

In [7], the constraint function is designed to control the input to zero as the distance between agents increases, and the value of the constraint function only depends on the distance between agents. The advantage of the constraint function compared to the potential energy function makes the control input bounded. As the number of agents in a multi-agent system increases, the rendezvous speed decreases exponentially. For example, in Figure.1, from $t-1$ to $t$, the control inputs of agent $i$ and agent $j$ decrease as the distance increases. However, for the agent $i$, it is necessary to move to the agent $j$ as soon as possible to prevent the disconnection of the topology connection.

Therefore, taking the agent $i$ as an example, the agent connection quality assessment algorithm is designed as follow:

TABLE 1. Connectivity Quality Assessment Algorithm

\begin{tabular}{|c|c|}
\hline Step1) & Initialize key location agent collection $M_{i}$, if $i \in M_{i}$, then agent $i$ is in a critical position \\
\hline Step2) & Calculate the set of neighbors common to agent $i$ at $t$ and $t$-1, i.e. $S=\left\{j \mid j \in N_{i}(t) \wedge j \in N_{i}(t-1), i \neq j\right\}$ \\
\hline Step3) & In the set $S$,finding the most distant agent $j$, i.e. $j=\arg \max \left\|x_{j}-x_{i}\right\|$ \\
\hline Step4) & Calculate the movement trends of Agent $i$ and agent $j$, i.e. $\left\|x_{i}(t)-x_{j}{ }^{\prime}(t)\right\|-\left\|x_{i}{ }^{\prime}(t)-x_{j}{ }^{\prime}(t)\right\|$ \\
\hline Step5) & $\begin{array}{l}\text { If }\left\|x_{i}(t)-x_{j}{ }^{\prime}(t)\right\|-\left\|x_{i}{ }^{\prime}(t)-x_{j}{ }^{\prime}(t)\right\|>0 \text { and }\left\|x_{j}-x_{i}\right\|>\rho \text {, then the agent is in a critical position and the } \\
\text { connection quality is poor, i.e. } i \in M_{i}\end{array}$ \\
\hline Step6) & $\begin{array}{c}\text { If }\left\|x_{j}-x_{i}\right\|>\rho \text { and }\left\|x_{i}(t)-x_{j}{ }^{\prime}(t)\right\|-\left\|x_{i}{ }^{\prime}(t)-x_{j}{ }^{\prime}(t)\right\|<0 \text {, then agent } i \text { has a tendency to move to agent } j, \\
\text { i.e. } i \notin M_{i}\end{array}$ \\
\hline
\end{tabular}

Based on the algorithm described in Table $1, \prod_{j \in N_{i}} \varphi_{i}\left(\left\|x_{j}-x_{i}\right\|\right)$ in formula (4) is represented as:

$$
\varphi_{i}(x)= \begin{cases}1, & i \notin M_{i} \\ 0.8, & i \in M_{i} \wedge x \in\left[0, \frac{4}{5} r\right) \\ -4 x+4, & i \in M_{i} \wedge x \in\left[\frac{4}{5} r, r\right)\end{cases}
$$

The advantage of this algorithm is that the traditional constraint function only depends on the distance between the agents, and the quality assessment algorithm is based on the direction of movement of the agent compared to the neighbor agent. If the agent is moving to other agents and the quality of the connection is good, there is no need to adjust its adjustment factor. It can therefore adapt to multi-agent systems with a greater number of agents. 


\section{SIMULATION}

The simulation experiments take a multi-agent system with 9 and 50 agents as examples to verify the effectiveness of the topology-based connectivity assessment strategy under extreme topology distribution and uniform topology distribution respectively.

\section{Simulation 1}

The simulation uses nine agents distributed in the plane as an example. The agent communication radius is 4 , the danger area is $\rho>3.2$, the adjustment factor is $\varepsilon=0.1$, and the distance between agents is close to the sensing radius.

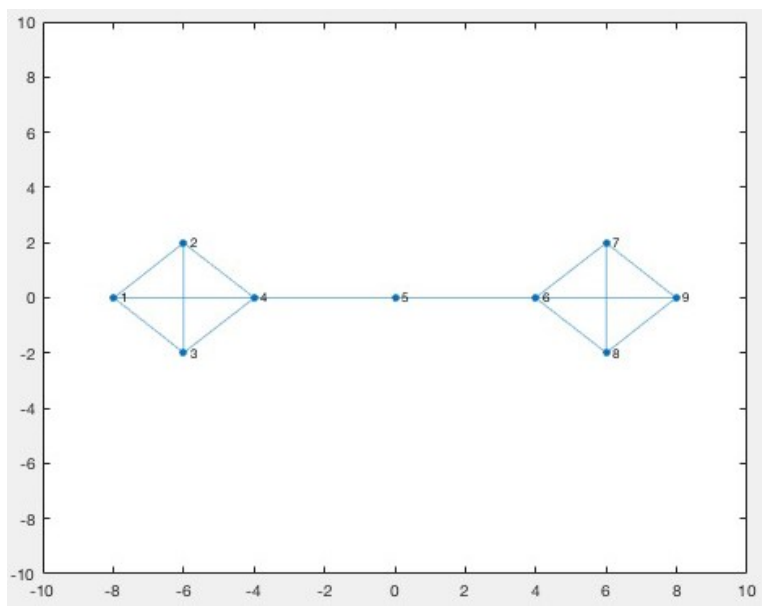

a) Initial network distribution

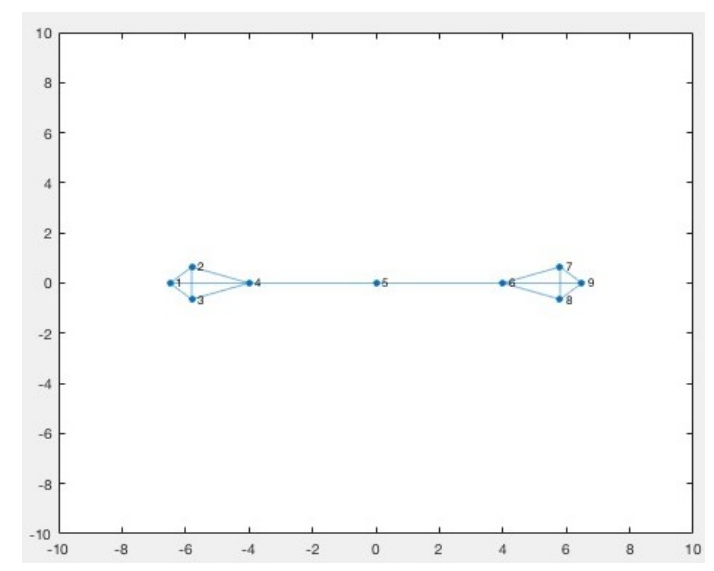

c) Quality assessment stategy

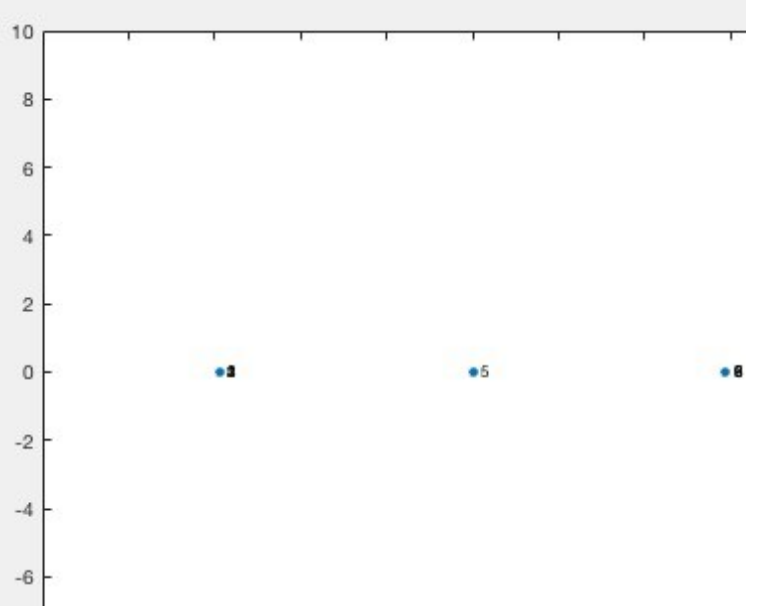

b) rendezvous by traditional stategy

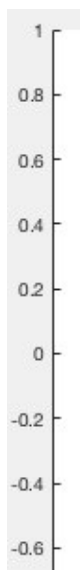

d) Final state

FIGURE 2. Agent's initial topology distribution and comparison with traditional aggregation strategy

As shown in Fig.2, compared with the traditional strategy (i.e. formula (3)), multi-agent rendezvous based on connectivity assessment can better preserve topology connectivity. 


\section{Simulation 2}

The simulation uses 50 multi-agents evenly distributed in a circular area with a radius of 3 as an example, in which the agent communication radius $r=1$, the danger area $\rho>0.8$, and the adjustment factor $\varepsilon=0.02$.

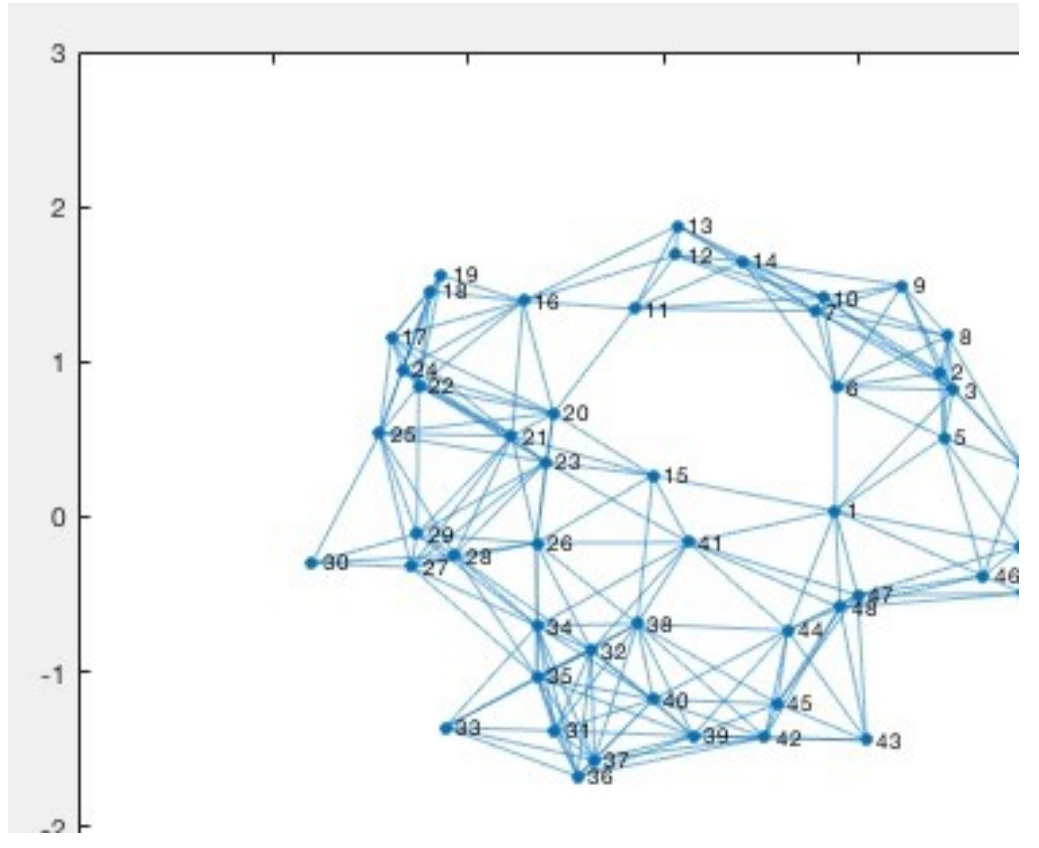

a) Initial network distribution

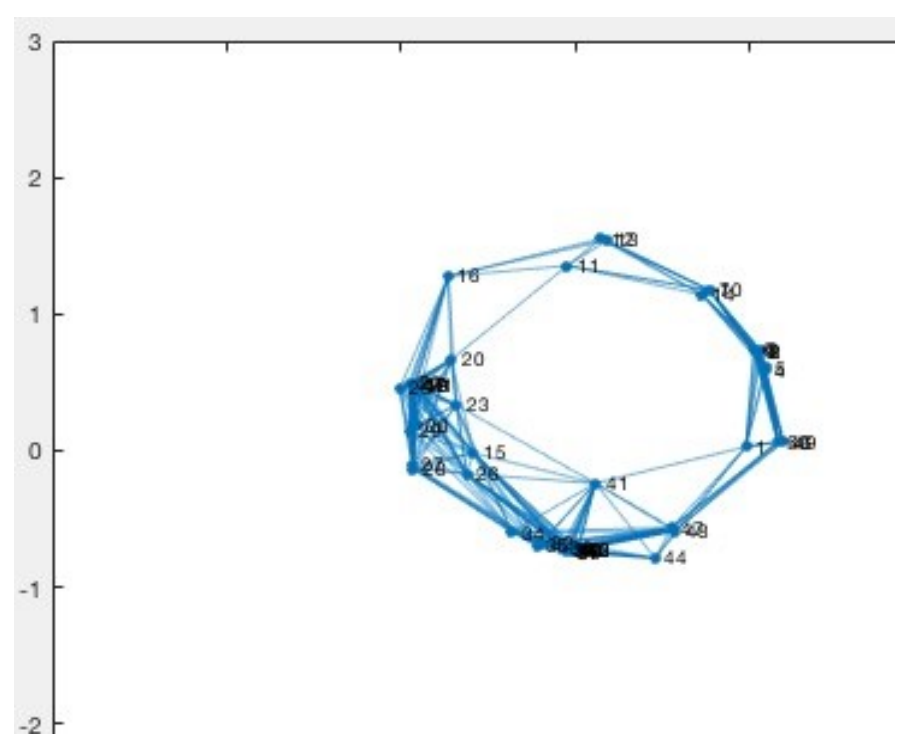

b) Agents are gathering 


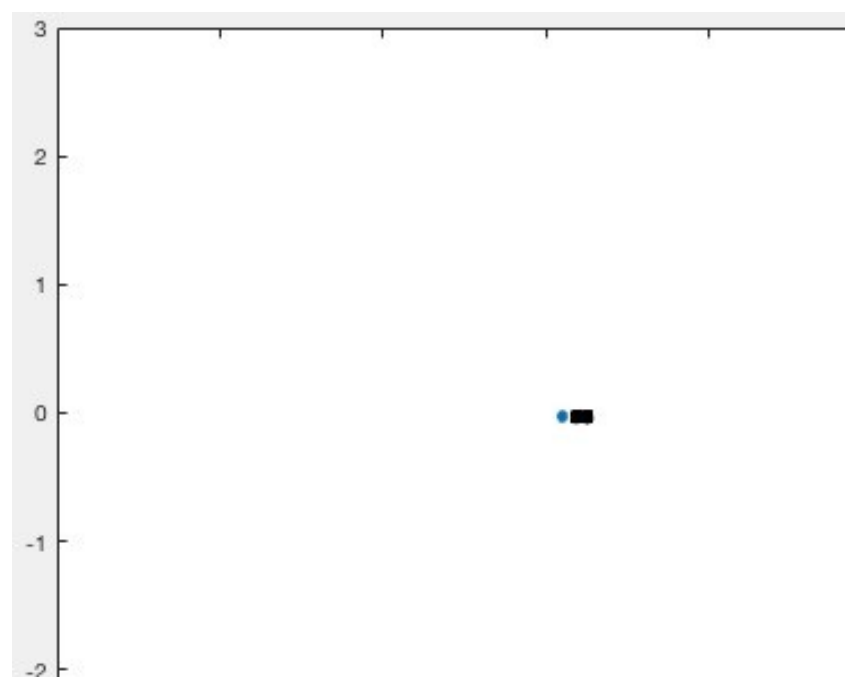

c) Final state

FIGURE 3. Multi-agent initial topology and rendezvous process

As shown in Fig. 4, under this strategy, multi-agents can achieve asymptotic rendezvous. When the agents have a tendency to break away from the communication range, the agents actively reduce the adjustment factor to achieve the purpose of preserving topology connectivity. Enable agents to rendezvous at the same point.

\section{CONCLUSION}

This paper mainly studies the multi-agent aggregation problem of preserving topology connectivity based on connectivity quality assessment. Based on the adjustment of the agent control input by the constraint function, the current connectivity of the agent is evaluated. Different from the original constraint function, under the strategy based on topological connectivity assessment, agents can roughly estimate the position and movement trend of the multi-agent in the system so that selecting more appropriate parameters to adjust the movement. The agents that are moving closer to other agents are unaffected by the unconstrained function, achieving progressive rendezvous that preserving topology connectivity.

\section{REFERENCES}

1. Ando H,Suzuki I,Yamashita M.Formation and agreement problems for synchronous mobile robots with limited visibility [C]//IEEE International Symposium on Intelligent Control.IEEE,1995:453-460.

2. Cortes J, Martinez S,Bullo F. Robust rendezvous for mobile autonomous agents via proximity graphs in arbitrary dimensions[J].IEEE Transactions on Automatic Control,2006, 51(8):1289-1298.

3. Spanos D P,Murray R M.Robust connectivity of networked vehicles[C]//Decision and Control, 2004.Cdc.IEEE Conference on.IEEE,2004:2893-2898 Vol.3.

4. Xiao F,Wang L,Chen T.Connectivity preservation for multi-agent rendezvous with link failure[J].Automatica,2012,48 (1):25-35.

5. Ji M, Egerstedt M. Distributed Coordination Control of Multiagent Systems While Preserving Connectedness[J]. IEEE Transactions on Robotics,2007,23(4):693-703.

6. Su H,Wang X,Chen G. Rendezvous of multiple mobile agents with preserved network connectivity[J].Systems \& Control Letters,2010,59(5):313-322.

7. Fan Y, Feng G, Gao Q. Bounded control for preserving connectivity of multi-agent systems using the constraint function approach[J]. Iet Control Theory \& Applications, 2012,6(11):1752-1757.

8. Lin J, Morse A S, Anderson B D O.The multi-agent rendezvous problem, part 1 the synchronous case [C]/Procofthe 43rd IEEE Conference on Decision and Control.2004: 1926-1931. 